\title{
A Dopant-Dependent Band Gap Narrowing Model Application for Bipolar Device Simulation
}

\author{
V. Palankovski, G. Kaiblinger-Grujin, H. Kosina, and S. Selberherr \\ Institute for Microelectronics, TU Vienna \\ Gusshausstr. 27-29, A-1040 Vienna, Austria
}

\begin{abstract}
We present a new band gap narrowing model which considers the semiconductor material and the dopant species for arbitrary finite temperatures. This unified treatment is especially useful for accurate device simulation. As a particular example we studied with our two-dimensional device simulator MINIMOS-NT the electrical behavior of a graded composition Si/SiGe HBT using a hydrodynamic transport model.
\end{abstract}

\section{Introduction}

Many papers were dedicated on band gap narrowing in semiconductors in the last 20 years (e.g. $[1,2]$ ). Despite of all, the optimal balance between accuracy of the results and simplicity of model implementation seems still not to be achieved. Commercial device simulators, such as ATLAS [3] , DESSIS [4], and MEDICI [5], use the logarithmic fit models for band gap narrowing in $\mathrm{Si}$ from $[1,2,6,7]$ which are simple to implement, but deliver unphysical values below and above definite doping levels. Furthermore, the functional form of models for $\mathrm{Si}$ is used for models for other materials (e.g. III-V compounds). Comparisons of these models are shown in Fig. 1. The physical limit our model offers $\left(0 \mathrm{meV}\right.$ at doping $\left.\mathrm{n}=0 \mathrm{~cm}^{-3}\right)$, the physically sound explanation of some existing effects it gives, and the simplicity of the model, make it very applicable for device simulation purposes.

\section{The New Model}

The basic assumption in our model is that band gap narrowing is a result of five types of many-body interactions (electron-electron, electron-impurity, hole-hole, holeimpurity, and electron-hole). At high doping concentrations we assume the electronimpurity contribution to be dominant. Though band gap narrowing is very difficult to be modeled rigorously due to the multiple carrier interactions one can approximate the energy shift by the classical self-energy of an ionized impurity interacting with an electron gas $[8]$.

$$
\Delta E_{g}=e \lim _{r \rightarrow 0}\left[V_{s}(r)-V(r)\right]
$$

Here $V_{s}(r)$ and $V(r)$ denote the screened and unscreened Coulomb potentials of the impurity, respectively. (1) represents the change in the electrostatic energy of the 


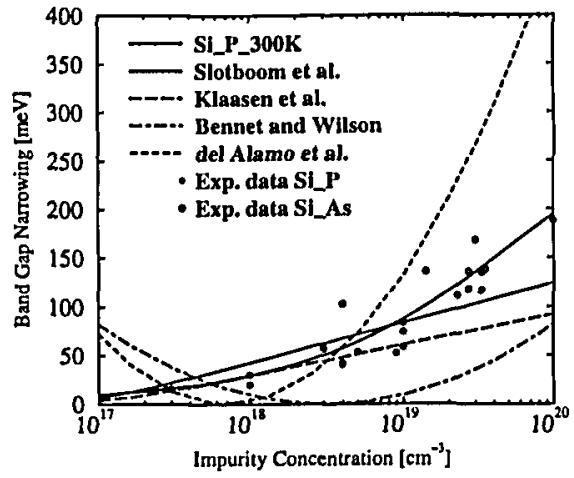

Figure 1:Comparison with models used in other device simulators.

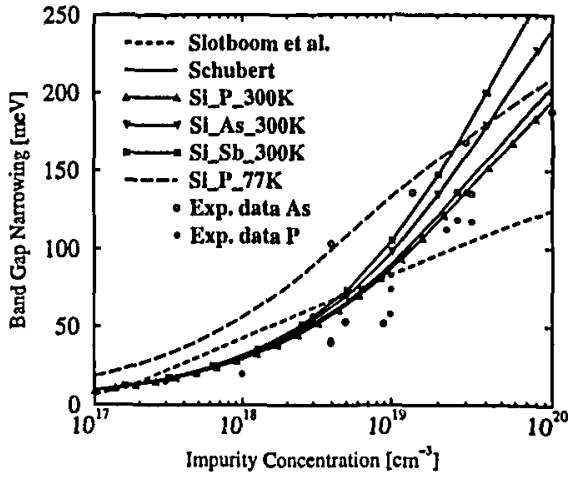

Figure 2: Influence of the dopant material on $\mathrm{BGN}$ in $\mathrm{n}-\mathrm{Si}$.

impurity before and after the electron gas redistribution. Assuming the potential of a point-like impurity gives a simple band gap narrowing model [8].

$$
\Delta E_{g}=-\frac{e^{2} \beta}{4 \pi \epsilon_{0} \epsilon_{r}} \quad \text { (2) } \quad \beta^{2}=\frac{n e^{2}}{\epsilon_{0} \epsilon_{r} k_{B} T} \cdot \frac{\mathcal{F}_{-1 / 2}(\eta)}{\mathcal{F}_{1 / 2}(\eta)}
$$

where $\beta$ denotes the inverse Thomas-Fermi length, and $\mathcal{F}_{j}(x)$ is the Fermi integral of order $j$ [9]. Removing the point-charge approximation yields a refined model. The charge density of the impurity can be accounted for by an atomic form factor $F$. Following the work [10] the impurity potential takes the form

$$
V(q)=\frac{e^{2}}{\epsilon_{0} \epsilon_{r}}\left(\frac{Z-F(q, \alpha)}{q^{2}+\beta^{2}}\right) \quad \text { (4) } \quad F(q, \alpha)=\frac{N \alpha^{2}}{q^{2}+\alpha^{2}}
$$

and after (1) leads to our final expression for the energy shift

$$
\Delta E_{g}=-\frac{e^{2} \beta}{4 \pi \epsilon_{0} \epsilon_{r}}\left[\frac{\beta N_{\mathrm{I}}}{\beta+\alpha_{\mathrm{I}}^{2}}-\frac{\beta N_{\mathrm{S} C}}{\beta+\alpha_{\mathrm{S} C}^{2}}+Z_{\mathrm{I}}-N_{\mathrm{I}}\right] \text {. }
$$

The subscripts $S C$ and $I$ refer to a semiconductor and impurity, respectively. $Z$ and $N$ are the atomic number and the number of electrons of a given material. $\alpha$ can be interpreted as size parameter of the electron charge density and $\alpha_{0}$ is the Bohr radius. They are expressed as

$$
\alpha=\frac{Z^{1 / 3}}{c_{k} \alpha_{0} \epsilon_{r}} \cdot \frac{1-2\left(\frac{Z}{N}\right)}{\frac{5}{3}-4\left(\frac{Z}{N}\right)^{1 / 3}} \quad \text { (7) } c_{k}=\frac{\Gamma(4 / 3)}{2}\left(\frac{3 \pi}{4}\right)^{2 / 3}\left(\frac{3}{5}\right)^{7 / 3} \approx 0.24 .
$$

The size parameter $\alpha$ uses $\epsilon_{r}=1$, which is the most pessimistic estimation, since it is still not clarified which value for $\epsilon_{r}$ in the range between 1 and $\epsilon_{S C}$ is valid at microscopic level. Even though the influence of dopant type is reduced to minimum this way, our model still delivers different results in agreement with experiment [11] (see Fig. 2). Note the stronger band gap narrowing at $77 \mathrm{~K}$ caused by higher degeneracy. Neglecting this effect results in an error of about $50 \%$. Our model gives also an answer to the question for the temperature dependency of the BGN in Fig. 3, i.e. it is not negligible at levels about $10^{10} \mathrm{~cm}^{-3}$. Thus, our band gap narrowing model is the first theoretically derived model predicting different shifts for various dopant species. 


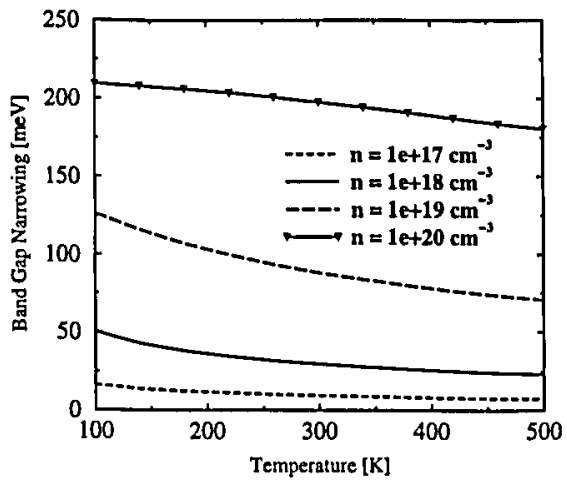

Figure 3: Temperature dependence of the band gap narrowing in phosphorus doped $\mathrm{Si}$.

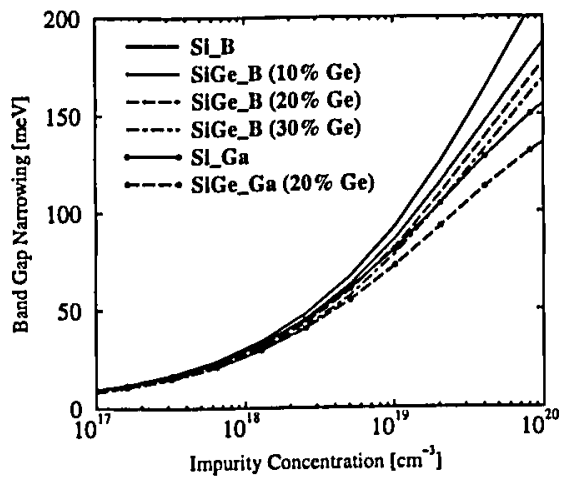

Figure 4: Influence of the dopant material and material composition in $\mathrm{p}$ SiGe.

\section{Extension of the Model to Compound Semiconductors}

Our model extends its validity also for compound semiconductors by material composition dependent relative DOS masses for electrons and holes, on one hand, and permittivity, on the other hand. The values used for the semiconductor electron number are calculated in a similar way. In Fig. 4 we present the results for boron and gallium doped SiGe for different Ge contents. The decrease of the BGN with increase of the Ge fraction was already experimentally observed [12]. Our theoretical approach explains this effect by the decreased valence density of states and increase of the relative permittivity in the SiGe alloy.

\section{Simulation Results}

As a particular example the electrical behavior of SiGe HBT was studied with our two-dimensional device simulator MINIMOS-NT [13] at different temperatures using a hydrodynamic transport model. Our investigations were performed in a comparative way for different dopant species and concentrations using the new models and the old ones. In Fig. 5 we present the Gummel plots for SiGe HBT at $77 \mathrm{~K}$ and $300 \mathrm{~K}$ obtained with the model of Slotboom et al. [1] (Mod. 1) and with our new model (Mod. 2). Note the significant difference in the current density values at $77 \mathrm{~K}$, resulting in higher current gain with the new model (Fig. 6), which is confirmed by experiments.

\section{Conclusion}

In summary, a new physically based analytical band gap narrowing model is presented. It accounts for different dopants and is applicable to compound semiconductors. It is in good agreement with the few available experimental data which exhibit an uncertainty of $10 \%$. In comparison with other existing models used for device simulation, the superiority of our new model is underlined. Finally, the important impact on the HBT device performance is studied.

\section{Acknowledgment}

This work is supported by Siemens AG, Munich, Germany. 


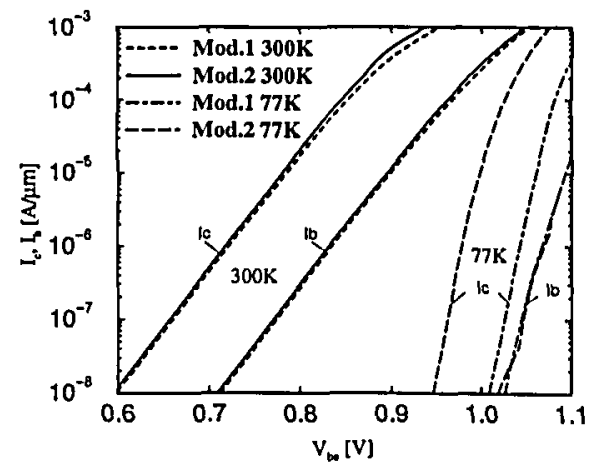

Figure 5: Gummel plots at $V_{c e}=2 \mathrm{~V}$ for Mod. 1 and Mod. 2.

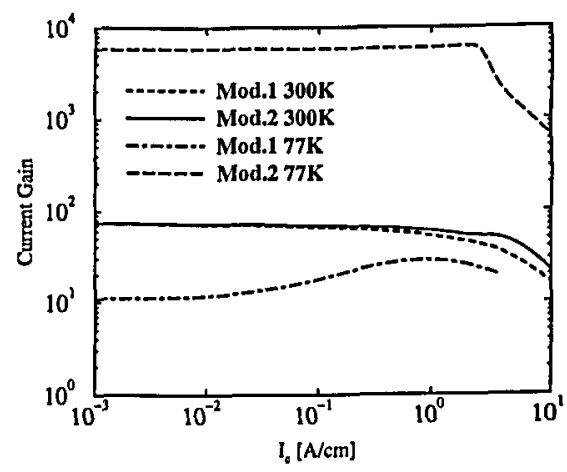

Figure 6: Current gain versus collector current for Mod. 1 and Mod. 2.

\section{References}

[1] J. Slotboom and H. de Graaff, "Measurements of Bandgap Narrowing in Si Bipolar Transistors," Solid-State Electron., vol. 19, pp. 857-862, 1976.

[2] D. Klaassen, J. Slotboom, and H. de Graaff, "Unified Apparent Bandgap Narrowing in $n$ - and $p$-Type Silicon," Solid-State Electron., vol. 35, no. 2, pp. 125-129, 1992.

[3] SILVACO International, Santa Clara, USA, ATLAS, User's Manual, Edition 2, Mar. 1994.

[4] ISE Integrated Systems Engineering AG, Zürich, Switzerland, DESSIS-ISE, ISE TCAD Release 5.0, Part 16, Mar. 1998.

[5] Technology Modeling Associates, Inc., Sunnyvale, California, TMA Medici, TwoDimensional Device Simulation Program, Version 4.0 User's Manual, Oct. 1997.

[6] J. DelAlamo, E. Swirhun, and R. Swanson, "Simultaneous measuring of hole lifetime, hole mobility and bandgap narrowing in heavily doped n-type Silicon," in Int. Electron Devices Meeting, pp. 290-293, 1985.

[7] H. Bennet and C.L.Wilson, "Statistical Comparisons of Data on Band-Gap Narrowing in Heavily Doped Silicon: Electrical and Optical Measurments," J.Appl.Phys., vol. 55, no. 10, pp. 3582-3587, 1984.

[8] E. Schubert, Doping in III-V Semiconductors. Cambridge University Press, 1993.

[9] D. Ferry, Semiconductors. New York: Macmillan, 1991.

[10] G. Kaiblinger-Grujin, H. Kosina, and S. Selberherr, "Influence of the Doping Element on the Electron Mobility in $n$-Silicon," J.Appl.Phys., vol. 83, no. 6, pp. 30963101, 1998.

[11] J.-S. Park, A. Neugroschel, and F. Lindholm, "Comments on Determination of Bandgap Narrowing from Activation Plots," IEEE Trans.Electron Devices, vol. 33, no. 7, pp. 1077-1078, 1986.

[12] Z. Matutinovic-Krstelj, V. Venkataraman, E. Prinz, J. Sturm, and C.W.Magee, "A Comprehensive Study of Lateral and Vertical Current Transport in Si/Si ${ }_{1-x} \mathrm{Ge}_{x} / \mathrm{Si}$ HBT's," in Int. Electron Devices Meeting, pp. 87-90, 1993.

[13] T. Simlinger, H. Brech, T. Grave, and S. Selberherr, "Simulation of Submicron Double-Heterojunction High Electron Mobility Transistors with MINIMOS-NT," IEEE Trans.Electron Devices, vol. 44, no. 5, pp. 700-707, 1997. 